IJBPAS, December, Special Issue, 2021, 10(12): 588-601

ISSN: 2277-4998

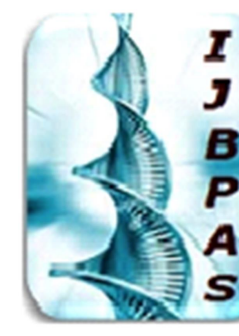

International Journal of Biology, Pharmacy and Allied Sciences (IJBPAS)

'A Bridge Betueen caboratory and QRendo'

www.ijbpas.com

\title{
IDENTIFICATION AND ANALYSIS OF THE SUITABLE ALGORITHM FOR ENERGY MINIMISATION OF PROTEIN
}

\author{
RITASHREE DASGUPTA \\ Department of Microbiology, Bose Institute, Kolkata
}

*Corresponding Author: Ritashree Dasgupta: E Mail: ritashreedasgupta@gmail.com

Received $19^{\text {th }}$ Aug. 2021; Revised $20^{\text {th }}$ Sept. 2021; Accepted $29^{\text {th }}$ Oct. 2021; Available online $1^{\text {st }}$ Dec. 2021

https://doi.org/10.31032/IJBPAS/2021/10.12.2052

\begin{abstract}
Aim -Earlier researches were unable to unfold an easy use of the existing energy minimisation algorithm that can be used to remove the stoichiometric clashes before MD simulation. The research aims to identify the effective and feasible energy minimisation algorithm that can be applied before MD simulation to conduct energy minimisation.

Methods- the protein with id 1A5Q is chosen from the RCSB-PDB database and the CHARMMM files are obtained. Based on them the energy minimisation of the protein is conducted in three different algorithms with respect to three different conditions (implicit, explicit and vacuum).
\end{abstract}

Results-SD is the fastest among three but $\mathrm{ABNR}$ reaches more $-\Delta \mathrm{G}$ whereas conjugate gradient minimises more but takes ample time.

Conclusion- A cocktail of SD and ABNR needs to be applied in case of energy minimisation.

Keywords: CHARMM, ABNR, SD, Conjugate gradient, MD simulation, GBSW,

GBMV, energy minimisation, protein ensemble, protein energy, electrostatic interactions, covalent bonds

\section{INTRODUCTION}

As per the opinion of Sliwoski et al. (2015) energy minimisation forms initial steps of molecular dynamic simulation. Before execution of the simulation it is of utmost necessity to minimise the energy of the protein to the least possible value $(\Delta \mathrm{G}=0)$. Along with that the protein needs to have a stable configuration with least steric 
clashes. This is because on simulation the protein structure automatically will experience distortions in structure. This in turn will elevate the energy of the protein. The main significance of the current research lies in this point that it delves and analyses the fittest algorithm that can be used for energy minimisation of protein in different environments. As per the research of Bhattacharya \& Cheng (2013) there is high degree of deviation between the structure of a native protein and a protein molecule studied computationally. Thus in this case refinement of the protein structures through energy minimisation is essential in order to reach the native structure of the protein. This research entails about a method of 3D refine which has a low CPU time and is based on the Royden-Fletcher-Goldfarb-Shannon (LBFGS) algorithm. On the contrary, this model was found to lack accuracy due to physical dynamism of the protein molecules. Additionally the research by Gainza et al. (2013) puts forth the application of protein redesign algorithm in the in-silico method with special description of the application of OSPREY. The application of OSPREY for protein designing has been found to be suitable but updated algorithms are needed for better modelling of proteins using this modelling suite. However all these approaches are difficult in terms of use and their application is limited to a narrower aspect of computational protein study.

Thus the main objective of the current research is finding out the most feasible algorithm for conducting the energy minimisation of the proteins for effective simulation of the protein. The objectives of the research are to undertake energy minimisation based onthree different algorithms and in three different environments (vacuum, explicit solvation and implicit solvation) and then finding out the ideal algorithm for conducting energy minimisation of protein.

\section{LITERATURE REVIEW}

2.1 Discussion on the different molecular interactions within a protein

Vanommeslaeghe et al. (2010) stated on a simpler note that the energy of a protein can be defined as a summation of the energy of the bonded and the non-bonded interactions in the protein as per the CHARMM force-field. The bonded interactions comprises of the stretching of the bonds and the bending of the bonding angle along with the torsional strain and the improper dihedral. It also contains the Urey-Bradley which is basically the energy involved in the interaction between the two atoms bonded by 2 bonds involving 3 atoms. Johnson et al. (2010) supported this and commented that the non-bonded 
interactions of a protein involve the van-der waals interaction between two amino acids which are non-bonded and the interaction is based in attraction and repulsive forces.

\subsection{Critical analysis of the significance of} energy minimisation in case of protein dynamic study

Energy minimisation can be termed as the technique to model the protein in a state where the energy of the protein is low. Energy minimisation leads to relaxation of the bonds leading to achievement of the local and in some cases global minima.

As per the study by Xu \& Zhang (2011) the importance of energy minimisation in case of protein modelling and study of protein dynamism is the refinement that is brought about in the protein backbone. As per the study of Bhattacharya \& Cheng (2013) in case of protein comparative modelling and structure prediction there is deviation from the original structure of the protein models because of the lack of accuracy of the models used in comparative modelling. The research by Zheng (2011) clearly supports these facts and elicits that there is poor structural quality of the models used for comparative modelling. In addition to this there are high steric clashes and torsional stress. Thus, for accurate comparative modelling it is necessary to refine the protein structure through conformational changes in the backbone structure of the protein.

\subsection{Analysis of the various algorithms for protein minimisation}

There are three different algorithms that are being used in energy minimisation namely steepest descent, conjugate gradient and the adapted Newton Raphson method. In case of energy minimisation using the steepest descent algorithm the local minima is reached. As per the comment of Svaiter (2018) the main principle of steepest descent is that the energy minimisation of the protein starts from the P0 and then based on the gradient descent method jumps from $\mathrm{Pi}$ to $\mathrm{Pi}+1$. The movement of the gradient is toward $-\Delta f(\mathrm{Pi})$. Gonzaga \& Schneider (2016) on the contrary argued that the main disadvantage related to the use of the steepest descent method is that the method might lead to formation of nonlinear lines and might not move towards the minimum energy efficiently.

As per the research by Yuan, Meng \& Li (2016) the main aim of the development of the conjugate gradient method is to avoid the deviations in the line search that occurs in case of steepest descent. This occurs by directing the new direction of movement so that it is dependent on the previous movement. Andrei (2014) supported this and stated that in each step a new point is based on the previous steps and based on 
those minimisation proceeds finding the minimum energy along the path.

Finally the adapted Newton Raphson method comes into play which can be termed as a costlier approach for energy minimisation. As per the opinion of Dutagaci, Heo \& Feig(2018) the ABNR is basically a combinatory approach of steepest descent and Newton Raphson method that is involved in correction of the geometries of the molecules in their subspace. Srivastava et al. (2015) at par with this commented that initially like the conjugate gradient the steepest descent is applied in this approach as well. Subsequent minimisation and gradient movement is conducted based on the steepest descent but in the last phase the method applies the Newton Raphson equations to correct the overall geometry displacement.

\section{METHODS}

The main work for the energy minimisation was done using the PDB structure of the P93A variant of the Bovine pancreatic ribonuclease $\mathrm{A}$. The pdb code is 1A5Q. The main advantage of using this structure was the replacement of proline with alanine in the 93 position of the protein without change in the stoichiometry of the protein imparting ease in studying the folding as well as unfolding of the protein molecule. The pdb structure of the protein was accessed from the RCSB data bank. Then in a LINUX platform the pdb structure of the protein was subjected to energy minimisation using three different algorithms in the CHARMM-GUI force field. The three different algorithms include the Steepest Descent (SD), Conjugate Gradient (CONJ) and Adapted NewtonRaphson Method (ABNR). The CHARMM-GUI force-field was chosen because although all the three force-fields are same but it is better suited to use CHARMM for such small proteins like ribonulcease.

The primary minimisation was done in vaccum. Later on the protein was subjected to a water box in the CHARMM-GUI force field and that structure was further energy minimised. The protein was also subjected to energy minimisation as per the implicit solvation model in the GBMV and GBSW model. Finally rather than using a single algorithm for energy minimisation of protein a 'cocktail' of algorithms were used to identify the best method for energy minimisation. The CHARMM-GUI used was available in the bioinformatics laboratory of Bose institute centenary building, Kolkata (CHARMM (Chemistry at Harvard Macromolecular Mechanics) Tutorial, 2018).

\section{RESULTS AND ANALYSIS}


1. Energy minimization of protein in vacuum

The energy minimisation of $1 \mathrm{~A} 5 \mathrm{Q}$ was carried out in vacuum for 7000 steps.

As per the result obtained in vacuum it can be seen that CPU time SD is the fastest but $\mathrm{SD}$ does not reach the minimum energy as compared to the other two. Rather it can be seen that CONJ and ABNR imparts a lowest energy configuration to the protein. However CONJ takes more time thus ABNR can be opted among one of the preferred algorithm for protein minimisation in vacuum. The minimisation is evident from the change in the position of the histidine 12 with an upward shift indicates the stoichiometric change in the protein structure as a result of the achievement of the minimised form.

\section{(Refer appendix 1)}

\section{Energy minimisation of protein after} explicit solvation

The 1A5Q was subjected to explicit solvation through establishing an interaction between the protein molecules and a water box. After explicit solvation energy minimisation using the three different algorithms was done for 4000 steps in the CHARMM force field (Refer appendix 2).

The results similar to that of energy minimisation in vacuum are observed in case of energy minimisation after explicit solvation. As compared to that of energy minimisation in vacuum, energy minimisation after explicit solvation is quite time consuming. This is because of the increase in the number of atoms due to the addition of the water molecules after the explicit solvation. It is seen that in case of 4000 steps were in the minimum time was taken by SD but the minimum as compared to the other two was not reached (Refer figure 5 and table 2 ). In addition the CONJ reached minimum energy for the protein but the time taken was too long. As compared to these two the ABNR seems to be more favourable as it reaches local minima and within a favourable amount of time. In addition there is a shift in Phenylalanine 8 after the explicit solvation and the energy minimisation.

\section{Energy minimisation of protein after implicit solvation}

In case of GBMV it can be seen that as compared to $\mathrm{SD}, \mathrm{ABNR}$ has lower energy value and on the other hand SD has taken more time. This can be implied as SD needed more time to reach global minima were as ABNR attained the global minima. In case of GBSW similar situation can be seen but SD took less time as compared to ABNR to complete the steps but has a higher energy value than ABNR indicating that SD needed more time to reach the 
global minima as compared to the reaching of the local minima by ABNR.

Inter-comparison between the two implicit solvation models it can be seen that GBSW is more efficient in reaching minimum energy as compared to GBSW as minimum energy is reached by GBSW at 4000 steps. However this difference in both the models based on the algorithms can be due to the simpler mathematical pathway used by the model (Refer appendix 3).

Table 1: CPU time and the energy of the protein in minimisation in vacuum

\begin{tabular}{|c|c|c|}
\hline Algorithm & CPU time & Energy \\
\hline SD & 1.02 mins & $-1700 J$ \\
\hline CONJ & 2.65 mins & $-1900 J<$ and $>-1800 J$ \\
\hline ABNR & 1.06 mins & $-1900 J<$ and $>-1800 J$ \\
\hline
\end{tabular}

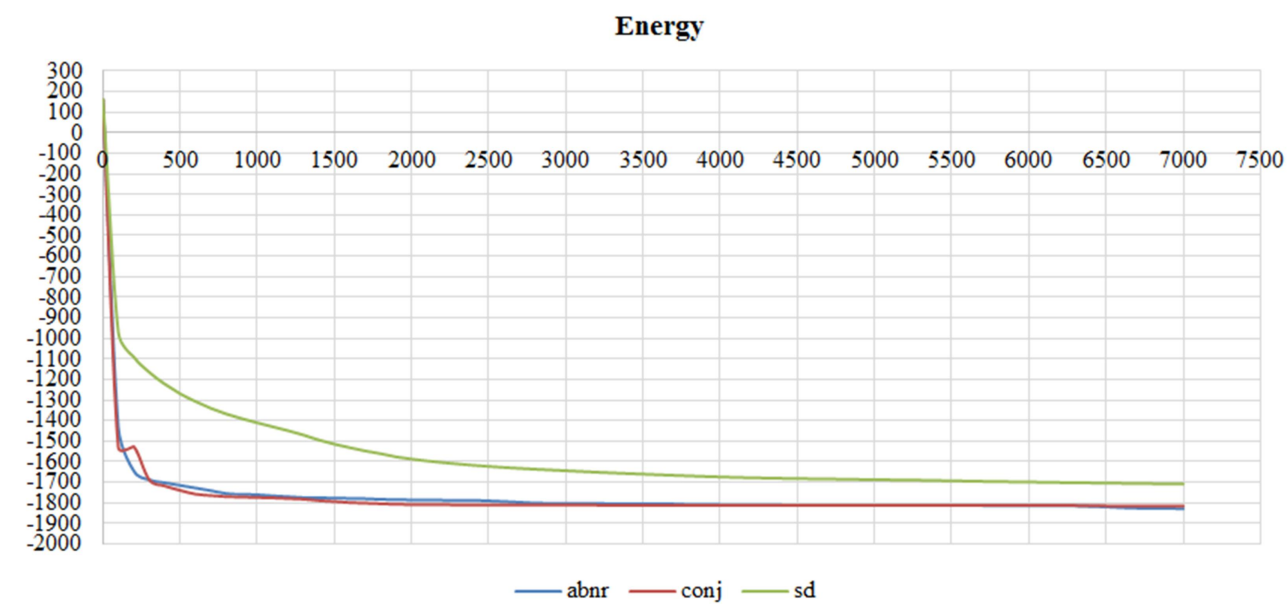

Figure 1: Total energy graph of protein for all three algorithms in case of minimisation in vacuum

Table 2: CPU time and minimised energy of protein in three different algorithms

\begin{tabular}{|c|c|c|}
\hline Algorithm & CPU time & Energy \\
\hline SD & $20.43 \mathrm{mins}$ & $<-100000 J$ \\
\hline CONJ & $\mathbf{5 6 . 4 3} \mathrm{mins}$ & $<-100500 J$ \\
\hline ABNR & $22.78 \mathrm{mins}$ & $<-100500 J$ \\
\hline
\end{tabular}

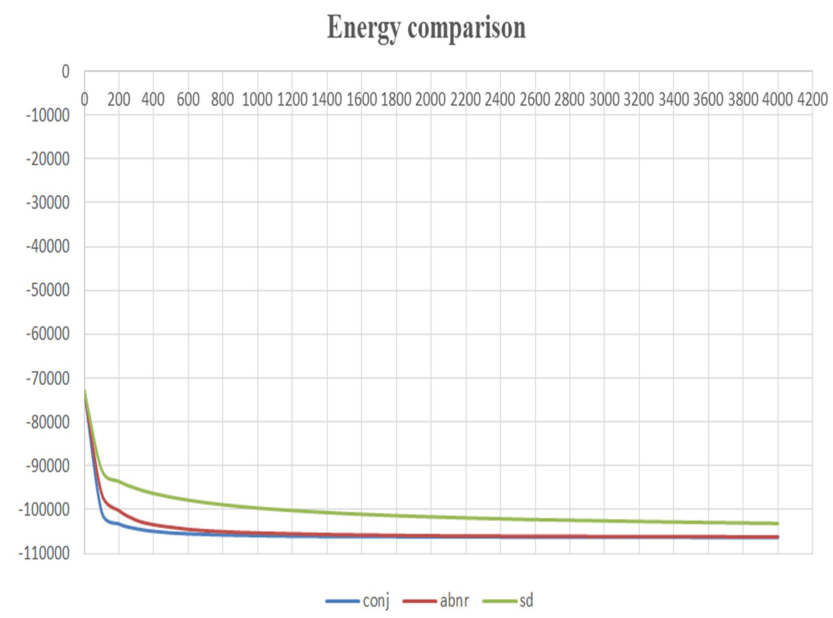

Figure 2: Energy of protein after energy minimisation by explicit solvation (increased time is due to the added water molecules) 


\section{DISCUSSION}

\section{Analysis for the better algorithm}

However a difference is observed in the SD and the ABNR based on the energy minimisation in both the explicit and vacuum based energy minimisation. SD keeps on improving the structure keeping in mind the minute changes in the bonding of each atom were as ABNR improvises the structure as a whole due to which the energy is minimised fast and to a larger extent. In case of SD at 4000 steps it is seen to be still improvising the protein model. However if the steps are increased then it is possible that SD might reach the minimum energy attained by CONJ and ABNR. This allows SD to take a lot more time to improvise the structure and minimise energy to a lesser extent in very small number of steps. Thus the inference that SD is the fastest among all might not be true. Rather it can be termed as the most effective among the three but and ABNR can be termed as the fastest among the three.

Thus it can be mentioned that SD reaches global minima (del $\mathrm{E}=0$ ) by checking all the available conformations. Whereas
$\mathrm{ABNR}$ reaches local minima $(\mathrm{del} \mathrm{E}=0)$ as it obtains minimum energy conformation corresponding to a particular area. In case of SD the step sizes are too small indicating the time consuming process and the ability to reach global minima. ABNR on the other hand due to taking large step size obtain convergence and thus is fats but obtains only local minima.

Finally it can be seen that both SD and ABNR are advantageous to energy minimisation. This is as per the research results by Morris, Deaven \& Ho (1996) that SD improvises a protein structure until the most stable conformation is reached. Were as in accordance to the research by Van Gunsteren \& Karplus (1980) ABNR takes less time and reaches a minimum energy conformation by making overall conformational changes and thus reaching local minima from which the protein cannot obtain another conformation. This result henceforth is also proved from the energy minimisation conducted. As each algorithm has their unique features a combination of different algorithms can give fruitful results. This was done in vacuum initially for 7000 steps to get the primary results. 


\section{Different combinations showing different values of del $\mathrm{E}$}

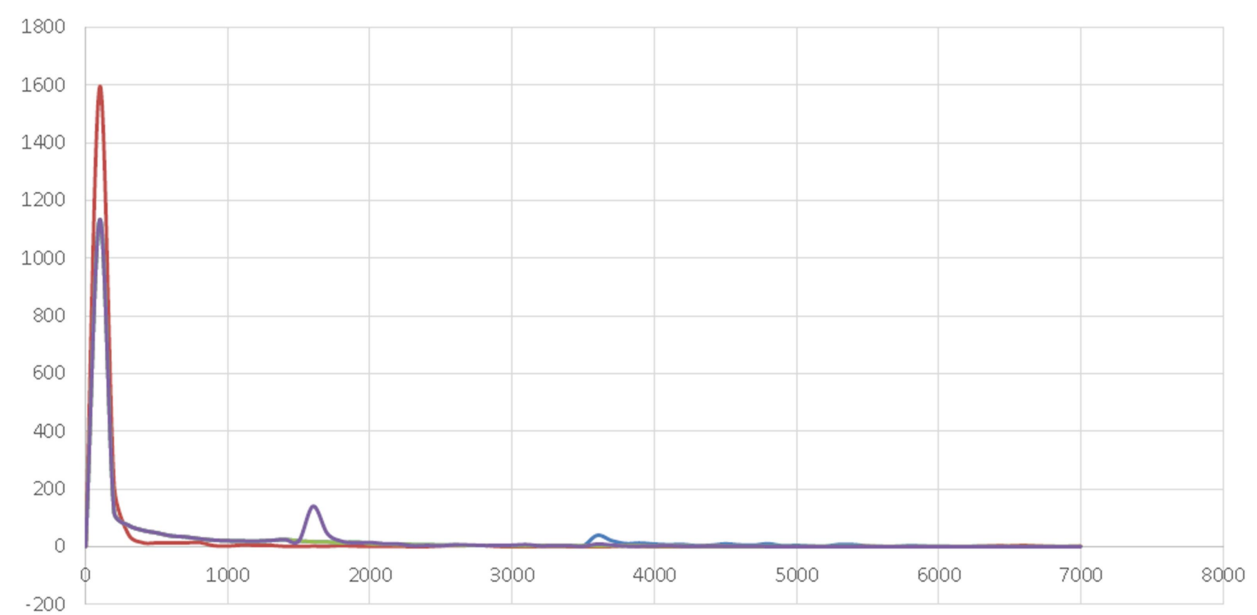

- delEsd-abnr —delE-abnr — delEsd —sd-abnr-sd

Figure 3: different values of del $E$ at different cominations of algorithm for energy minimisation indicating that delE for sd-abnr-sd is least and tends to 0

Table 3: delE values for different combination of algorithms that were tested indicating that as-abnr-sd reaches

\begin{tabular}{|c|c|c|c|c|}
\hline \multicolumn{6}{|c|}{ delE $=0$ faster in 7000 steps through both global and local minimisation } \\
\hline cycles & delE-abnr & delEsd & delEsd-abnr & deEsd-abnr-sd \\
\hline 7000 & 1.67987 & 0.9165 & 0.22591 & 0.00058 \\
\hline
\end{tabular}

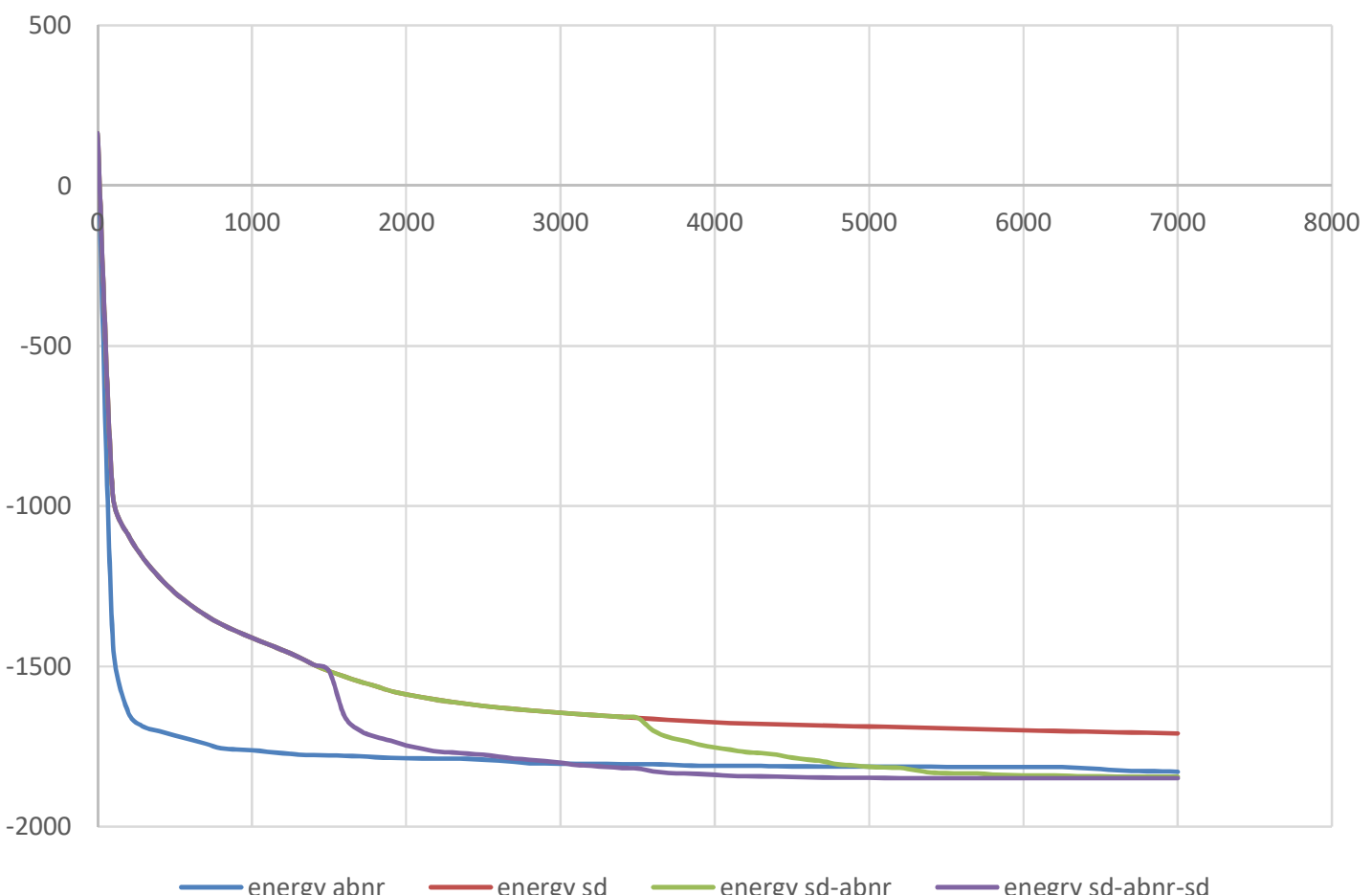

Figure 4: Graph showing the energy curves for each of the algorithm set. This indicates that sd-abnr-sd has the minimum gibbs free energy among all indicating its suitability for energy minimisation 


\section{CONCLUSION}

The most favourable among the combinations of SD and ABNR are SDABNR-SD. The minimum energy reached by this combination is del $\mathrm{E}=0.00058$ indicating that the value tends to reach zero at 7000 steps. The energy value indicates that it has reached stalest configuration as there are not further exchanges in the conformation and the energy value is constant at -1848.7 from 6800 to 7000 steps. That time that it took was 1.05 mins. This combination can be taken as a better option because initially using SD will lead to removal of all the steric clashes and the bulky group and achieving a stable structure. Then further energy minimisation with ABNR will lead to improvement in the overall structure of the protein. Final energy minimisation with SD will lead to refinement of the internal stoichiometric distortions thus leading to reaching of global minima. Initial 2000 steps of SD then 4000 steps of ABNR and final 2000 steps of SD will be executed to minimise the protein.

\section{LIMITATIONS AND FUTURE} SCOPE

Due to time constraint in case of explicit solvation 7000 steps were not done. In addition the final structure of the protein based on the SD-ABNR-SD model was minimised in vacuum and further minimisation in explicit and implicit solvation model needed to be done. Due to lack of time this analysis was not done and this holds a future prospect for further research. In addition CONJ although efficient but being an unfavourable algorithm was not further utilised into the combinational approach. This is one loophole of the research which can be rectified with further research on it.

\section{Acknowledgements}

Primarily I want to acknowledge my gratitude to Dr.Subhra Ghosh Dastidar, Bose Institute, Kolkata for guiding me and allowing me to do this project. I want to extend my gratefulness to the institute for letting me use their software and the research related facilities for doing the project.

\section{REFERENCE}

[1] Andrei, N. (2014). An accelerated subspace minimization three-term conjugate gradient algorithm for unconstrained optimization. Numerical Algorithms, 65(4), 859-874.

[2] Baker, C. M. (2015). Polarizable force fields for molecular dynamics simulations of biomolecules. Wiley Interdisciplinary Reviews: Computational Molecular Science,5(2), 241-254. 
[3] Bhattacharya, D., \& Cheng, J. (2013). 3Drefine: Consistent protein structure refinement by optimizing hydrogen bonding network and atomic-level energy minimization. Proteins: Structure, Function, and Bioinformatics, 81(1), 119-131.

[4] CHARMM (Chemistry at HARvard Macromolecular Mechanics) Tutorial. charmmtutorial.org. retrieved on: $5^{\text {th }}$ june 2015.retrieved from: https://www.charmmtutorial.org/ind ex.php/CHARMM_Tutorial

[5] Cino, E. A., Choy, W. Y., \& Karttunen, M. (2012). Comparison of secondary structure formation using 10 different force fields in microsecond molecular dynamics simulations. Journal of chemical theory and computation, 8(8), 27252740.

[6] Dutagaci, B., Heo, L., \& Feig, M. (2018). Structure refinement of membrane proteins via molecular dynamics simulations. Proteins: Structure, Function, and Bioinformatics.

[7] Gainza, P., Roberts, K. E., Georgiev, I., Lilien, R. H., Keedy, D. A., Chen, C. Y., \& Donald, B. R. (2013). OSPREY: protein design with ensembles, flexibility, and provable algorithms. In Methods in enzymology, 523,87-107. Academic Press.

[8] Galindo-Murillo, R., Robertson, J. C., Zgarbová, M., Sponer, J., Otyepka, M., Jurečka, P., \& Cheatham III, T. E. (2016). Assessing the current state of AMBER force field modifications for DNA. Journal of chemical theory and computation, 12(8), 4114-4127.

[9] Gonzaga, C. C., \& Schneider, R. M. (2016). On the steepest descent algorithm for quadratic functions. Computational

Optimization and Applications, 63(2), 523-542.

[10] Johnson, E. R., Keinan, S., MoriSanchez, P., Contreras-Garcia, J., Cohen, A. J., \& Yang, W. (2010). Revealing noncovalent interactions. Journal of the American Chemical Society, 132(18), 64986506.

[11] Jones, D. T., Singh, T., Kosciolek, T., \& Tetchner, S. (2014). MetaPSICOV: combining coevolution methods for accurate prediction of contacts and long range hydrogen bonding in proteins. Bioinformatics, 31(7), 999-1006. 
[12] Lynch, I., \& Dawson, K. A. (2008). Protein-nanoparticle interactions. Nano today, 3(1-2), 40-47.

[13] Morris, J. R., Deaven, D. M., \&Ho, K. M. (1996). Geneticalgorithm energy minimization for point charges on a sphere. Physical Review B, 53(4), R1740.

[14] Onufriev, A. (2010). The generalized Born model: its foundation, applications, and limitations. Departments of Computer Science and Physics, Blacksburg, Virginia, USA.

[15] Piana, S., Klepeis, J. L., \& Shaw, D. E. (2014). Assessing the accuracy of physical models used in protein-folding simulations: quantitative evidence from long molecular dynamics simulations. Current opinion in structural biology, 24, 98-105.

[16] Pu, M., Garrahan, J. P., \&Hirst, J. D. (2011). Comparison of implicit solvent models and force fields in molecular dynamics simulations of the PB1 domain. Chemical Physics Letters, 515(4-6), 283289.

[17] Sarzyńska, J., Mickiewicz, A., Miłostan, M., Łukasiak, P., Błażewicz, J., Figlerowicz, M.,
\&Kuliński, T. (2010). Flexibility of dicer studied by implicit solvent molecular dynamics simulations. Computational Methods in Science and Technology, 16(1), 97104.

[18] Sliwoski, G., Kothiwale, S., Meiler, J., \& Lowe, E. W. (2014). Computational methods in drug discovery. Pharmacological reviews, 66(1), 334-395.

[19] Srivastava, J. K., Awatade, N. T., Bhat, H. R., Kmit, A., Mendes, K., Ramos, M., \& Singh, U. P. (2015). Pharmacological evaluation of hybrid thiazolidin-4-one-1, 3, 5triazines for $\mathrm{NF}-\kappa \mathrm{B}$, biofilm and CFTR activity. RSC Advances, 5(108), 88710-88718.

[20] Standard, J. M. (2015). Energy minimization methods. Chemistry, 380. (SIMILAR RESEARCH)

[21] Svaiter, B. F. (2018). Holder continuity of the steepest descent direction for multiobjective optimization. arXiv preprint arXiv:1802.01402.

[22] Van Gunsteren, W. F., \&Karplus, M. (1980). A method for constrained energy minimization of macromolecules. Journal of Computational Chemistry, 1(3), 266-274. 
[23] Vanommeslaeghe, K., Hatcher, E., Acharya, C., Kundu, S., Zhong, S., Shim, J., \& MackerellJr, A. D. (2010). CHARMM general force field: A force field for drug-like molecules compatible with the CHARMM all-atom additive biological force fields. Journal of computational chemistry,31(4), 671-690.

[24] Xu, D., \& Zhang, Y. (2011). Improving the physical realism and structural accuracy of protein models by a two-step atomic-level energy minimization. Biophysical journal, 101(10), 2525-2534.

[25] Xu, Y., Vanommeslaeghe, K., Aleksandrov, A., MacKerellJr, A. D., \& Nilsson, L. (2016). Additive CHARMM force field for naturally occurring modified ribonucleotides. Journal of computational chemistry, 37(10), 896-912.

[26] Yuan, G., Meng, Z., \& Li, Y. (2016). A modified Hestenes and Stiefel conjugate gradient algorithm for large-scale nonsmooth minimizations and nonlinear equations. Journal of Optimization Theory and Applications, 168(1), 129-152.

[27] Zheng, W. (2011). Accurate flexible fitting of high-resolution protein structures into cryoelectron microscopy maps using coarse-grained pseudo-energy minimization. Biophysical journal, 100(2), 478-488. 


\section{Appendices}

Appendix 1: structural changes in the ribonuclease secondary structure after the minimisation that can be owed to the shift in the amino-acid positions

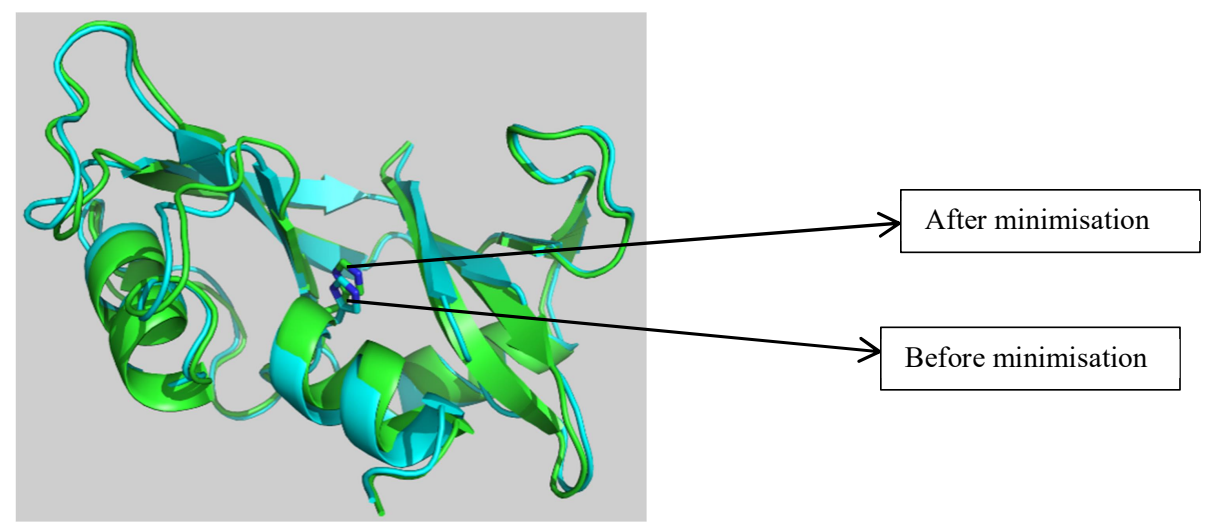

Appendix 2: Ribonuclease within a water box in explicit solvation in CHARMM-GUI

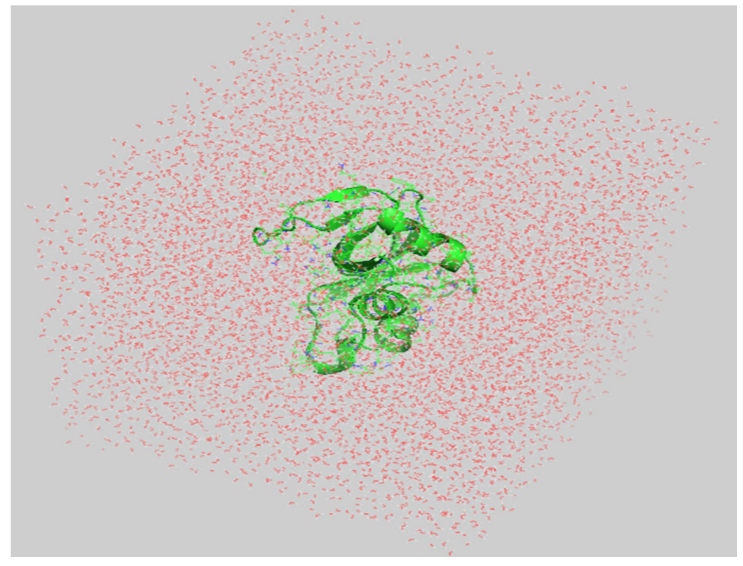


Appendix 3: Structural changes in protein side chain after explicit solvation (there is shift in the position after minimisation thus leading to removal of steric clashes)

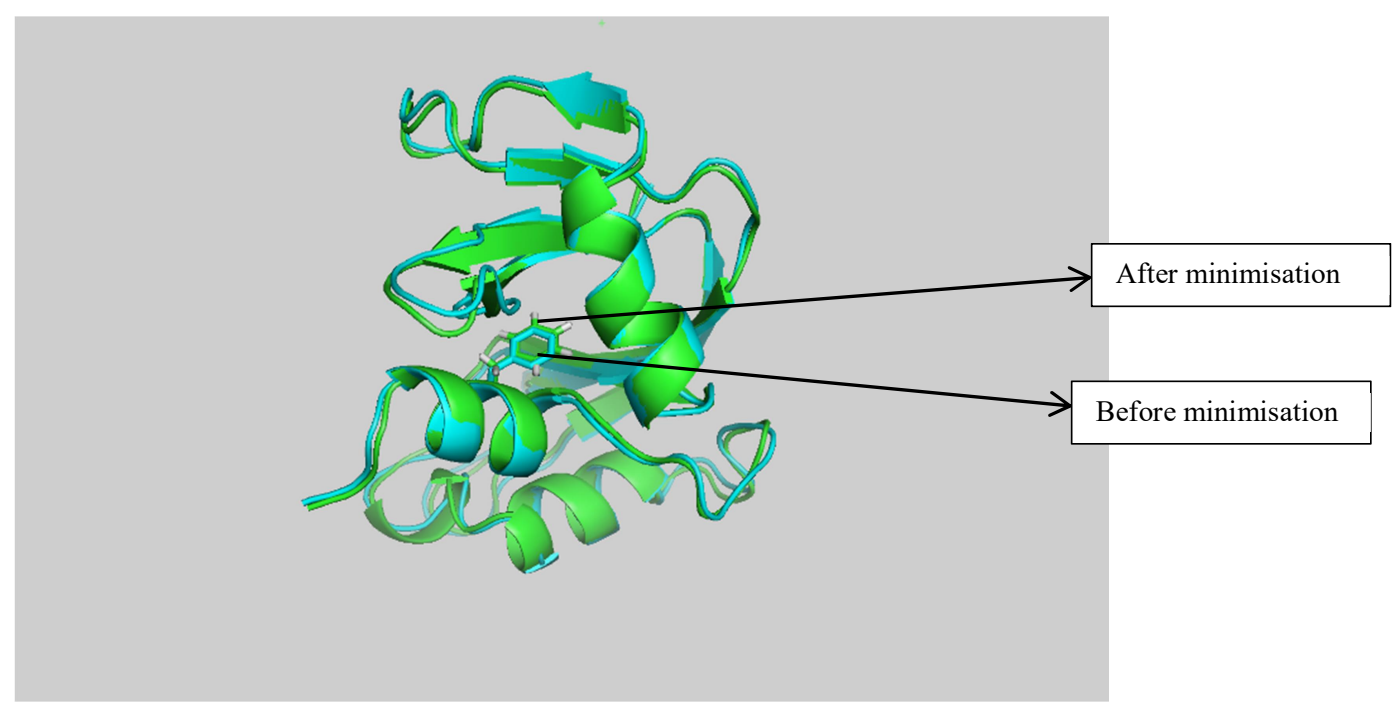

